\title{
La calidad del empleo en las residencias para mayores: incidencia en la gestión de la covid-19
}

\author{
Julia Montserrat Codorniu \\ Consultora, Red Europea de Protección Social \\ jmontserratc@gmail.com
}

\begin{abstract}
COVID-19aren eztandak adineko pertsonentzako egoitza-zerbitzuen kudeaketan akats ugari daudela ohartaraztea nabarmendu du. Osasun-larrialdiko egoera honetan, begi-bistakoak izan dira lanprekarizazioaren maila handia eta Administrazio Publikoak zentro horietako arreta pertsonalaren kalitatea ziurtatzeko dituen akatsak edo kontrolmekanismo eskasak. Artikulu honetan, adin handikoentzako egoitza-zerbitzuetako lanprekarietatea eta langile-hornidura osatzen duten askotariko faktoreak aztertzen dira, betiere erakunde pribatuetan zerbitzu publikoak gero eta kanpora ateratzen eta egoitza-plazen eskaintza gero eta handiagoa den testuinguruan. Emaitzak 20122018 aldiko bilakaeraren ikuspegitik azaltzen dira eta Espainiako eskalan, autonomia-erkidegoek berariazko erakusten dituztenei erreparatu gabe.
\end{abstract}

\section{GAKO-HITZAK:}

Adinekoen egoitzak, enplegua, kontrola, soldatak, arta-kalitatea.
El estallido de la covid-19 ha alertado de numerosas deficiencias en la gestión de los servicios residenciales para personas mayores. El elevado grado de precarización laboral y los fallos o insuficientes mecanismos de control de la Administración Pública para asegurar la calidad de la atención personal en dichos centros se han hecho evidentes en esta situación de emergencia sanitaria. Este artículo analiza los diversos factores que conforman la precariedad laboral de los servicios residenciales para mayores y la dotación de personal en el contexto de creciente externalización de los servicios públicos en organizaciones privadas y de aumento de la oferta de plazas residenciales. Los resultados se muestran desde la perspectiva de su evolución en el periodo 2012-2018 y a escala del conjunto de España, sin detenerse en las particulares que presenta cada una de las comunidades autónomas.

\section{Palabras Clave:}

Residencias mayores, empleo, control, salarios, calidad atención. 


\section{La oferta de los servicios residenciales para mayores}

Este apartado trata de poner en relación el crecimiento de plazas residenciales para mayores en los últimos años con indicadores demográficos, económicos y de políticas públicas que puedan haber influido en su evolución.

La primera constatación es que no existe una estadística "única” de centros y plazas residenciales para mayores, lo cual obliga a buscar en diferentes fuentes de información; no obstante, las cifras obtenidas, sin que coincidan exactamente entre ellas, son bastante similares. Las diferencias pueden ser debidas a la inclusión o no de otro tipo de alojamiento residencial como las viviendas para mayores, la consideración o no de algún tipo organización no lucrativa, o la diferencia en las fechas de recogida de los datos.

El Cuadro 1 ofrece información sobre la evolución de las plazas residenciales para mayores y de diversos factores demográficos y económicos que influencian el crecimiento de su demanda. Se toma como referencia del número total de plazas la cifra ofrecida por el Imserso para 2018 y las cifras del CSIC para los años anteriores. El desglose entre plazas públicas y plazas privadas se realiza según el concepto de "financiación”, es decir, "quién es el responsable de pagar la plaza”, diferenciando plazas de responsabilidad pública ${ }^{1}$ - financiación pública-y plazas privadas. No se incluyen como plazas financiadas públicamente las "prestaciones vinculadas al servicio” ya que la Administración realiza una transferencia monetaria a la persona beneficiaria para ayudarla a pagar el precio de una plaza en una residencia privada, no estableciendo una relación directa con el centro; en otras palabras, la Administración no es el "cliente".
Según las estimaciones realizadas con los criterios señalados en el párrafo anterior, se obtiene que un $44,4 \%$ de las plazas en residencias de mayores son de financiación pública. Sin embargo, el informe del Imserso señala un porcentaje mayor de plazas públicas, el 59,4\%, lo cual indica que posiblemente incluye las prestaciones vinculadas a servicio.

La oferta de servicios residenciales para mayores muestra un crecimiento importante, aproximadamente un 44,2\% entre 2005 y 2018 , alcanzando un total de 381.158 plazas. Ello significa pasar de una cobertura de 3,7 a 4,2 por cada 100 habitantes de 65 y más años. Las causas del rápido crecimiento de la oferta residencial para mayores hay que buscarlas en el conjunto de factores sociales, demográficos, económicos y políticas públicas acaecidos en este periodo. El entorno familiar cambia su estructura tradicional; los padres ya no viven con los hijos y, a menudo, tampoco viven en el mismo municipio, dando lugar a un aumento del número de hogares formados por una pareja o unifamiliares con edades de más de 65 años. Las mujeres aumentan su participación en el mercado laboral y el "prototipo" de hijas/nueras cuidadoras se diluye.

La esperanza de vida de la población a los 65 años crece sin cesar. En trece años ha aumentado casi dos puntos porcentuales, alcanzando en 2018 los 19,24 años en los hombres y los 23,09 años en las mujeres. Ello incide en un incremento de la ratio de envejecimiento ${ }^{2}$ y aumenta con mayor intensidad en el segmento de población de 80 y más años. Las proyecciones demográficas muestran un crecimiento continuado y se estima que, en 2050, más de un tercio de la población (37,5\%) tendrá más de 65 años y más de la mitad de ellos (57,2\%) tendrá más de 80 años.

Cuadro 1. Oferta de plazas residenciales para mayores y otros indicadores (España)

\begin{tabular}{|c|c|c|c|c|c|c|c|c|c|}
\hline Años & $\begin{array}{c}\text { Número } \\
\text { total de } \\
\text { plazas }\end{array}$ & $\begin{array}{l}\text { Plazas con } \\
\text { financiación } \\
\text { pública (\%) }\end{array}$ & $\begin{array}{l}\text { Plazas con } \\
\text { financiación } \\
\text { privada (\%) }\end{array}$ & $\begin{array}{c}\text { Porcentaje } \\
\text { población } \\
\text { de } 65 \text { y más } \\
\text { años }\end{array}$ & $\begin{array}{l}\text { Porcentaje } \\
\text { población } \\
\text { de } 80 \text { y } \\
\text { más años }\end{array}$ & $\begin{array}{l}\text { Pensión } \\
\text { media } \\
\text { jubilación. } \\
\text { Reg. general } \\
\text { (euros/mes) }\end{array}$ & $\begin{array}{l}\text { Valor } \\
\text { mediano } \\
\text { activos } \\
\text { (miles } \\
\text { euros) }\end{array}$ & $\begin{array}{c}\text { Tenencia } \\
\text { vivienda } \\
\text { principal } \\
\text { (\% jubilados) }\end{array}$ & $\begin{array}{c}\text { Tenencia } \\
\text { otros activos } \\
\text { inmobiliarios } \\
\text { (\%) }\end{array}$ \\
\hline 2005 & 271.801 & $\mathrm{~N} / \mathrm{C}$ & $\mathrm{N} / \mathrm{C}$ & 16,62 & 4,31 & 868,40 & 203,1 & 87,9 & 38,9 \\
\hline 2008 & 308.930 & 26,8 & 73,2 & 16,54 & 4,60 & $1.029,95$ & 229,2 & 90,7 & 43,2 \\
\hline 2011 & $344 \cdot 310$ & 37,5 & 62,5 & 17,15 & 5,09 & $1.145,28$ & 208,7 & 88,8 & 46,6 \\
\hline 2014 & 359.035 & 38,1 & 61,9 & 18,05 & 5,67 & $1.152,28$ & 189,7 & 90,4 & 52,9 \\
\hline 2017 & 366.633 & 44,0 & 56,0 & 18,82 & 6,11 & $1.216,54$ & 178,4 & 85,5 & 57,7 \\
\hline 2018 & 381.158 & 44,4 & 55,6 & 19,07 & 6,15 & $1.252,66$ & Pendiente & Pendiente & Pendiente \\
\hline
\end{tabular}

Fuente: Elaboración propia.

${ }^{1}$ Se realiza una aproximación con los datos del SISAAD, calculando la media anual de usuarios de las personas beneficiarias de la prestación.
${ }^{2}$ Se mide como la población de más de 65 años sobre la población total. 
El aumento de años de vida va ligado frecuentemente a pluripatologías físicas y psíquicas que aumentan las discapacidades de las personas. En 2006, la Encuesta sobre discapacidades, deficiencias y estado de salud (EDDES, 1999) cifraba en 2,07 millones las personas de 65 años y más con algún tipo de discapacidades. Aunque no hay una correlación cierta entre estado de salud y edad, la evidencia muestra una propensión a aumentar la discapacidad a medida que aumenta la edad.

La situación económica de las personas jubiladas es uno de los factores más relevantes en las decisiones de las empresas proveedoras de servicios residenciales para mayores. La pensión media de jubilación de la seguridad social -régimen generalcrece progresivamente desde 2005 (año del dato) hasta nuestros días. Si bien la pensión de jubilación ha aumentado en dicho periodo un 44,2 \% (media interanual del 3,4\%), el factor más importante es la riqueza patrimonial de las personas jubiladas, con especial incidencia en la tenencia de la vivienda habitual, así como la tenencia de otros activos financieros.

Es obvio que más de la mitad de los pensionistas no podrían pagar el precio de una residencia 3 solo con el importe de la pensión pública, pero el hecho de disponer de un patrimonio hace que este pueda ser utilizado como suplemento financiero para cubrir el precio de la plaza en una residencia y, también, se utiliza para complementar el copago de una plaza de responsabilidad pública según la Ley de Promoción para la Autonomía Personal y Atención a la Dependencia (LAPAD).

La encuesta financiera a las familias realizada por el Banco de España cada tres años (se han escogido los años del cuadro de acuerdo con los años de la encuesta) muestra entre sus resultados el desglose de renta y riqueza de los hogares de las personas cuyo cabeza de familia es un jubilado. El valor del patrimonio que figura en la estadística es solo un valor de referencia, ya que no es el valor real de mercado debido a que está calculado según criterios de Hacienda ("valor catastral"), pero es significativo que dicho valor sea superior a la mediana de todos los hogares. Otro dato importante es que más de un $85 \%$ de este segmento de población es propietario de su vivienda habitual y que casi un $60 \%$ posee otros activos financieros (segundas viviendas, pisos, locales o plazas de garaje, entre otros) los cuales pueden proporcionar una renta suplementaria a la pensión, o bien son susceptibles de ser convertidos en liquidez en caso de necesidad. En la evolución del valor del patrimonio se puede percibir el efecto de la crisis económica del 2008, la cual incidió en una disminución de la riqueza de los jubilados.

3 Según diversas webs consultadas, el abanico de precio en las residencias privadas es muy amplio pudiendo oscilar entre 1.500 a 3.000 euros o más, dependiendo de muchos factores (instalaciones, ubicación geográfica o tipo de actividades, entre otros).
La oferta "privada" de plazas en residencias para mayores es alentada no solo por los factores demográficos, familiares y la relativa buena salud económica de los potenciales “clientes”, sino también por la importante y creciente demanda de plazas del sector público para ofrecerla a las personas beneficiarios de la LAPAD. Se observa en el Cuadro 1 que, en 2018, casi la mitad de las plazas residenciales son de provisión pública, es decir, aquellas que la Administración tiene obligación de proveer a las personas en situación de dependencia reconocidas según la ley, de las cuales más de un noventa por ciento, aproximadamente, son gestionadas por empresas privadas según las estimaciones realizadas en este estudio.

La importante financiación pública de plazas en residencias privadas constituye un buen colchón económico para el sector privado, ya que, aunque el precio plaza acordado en el concierto pueda ser inferior al precio de una plaza privada, les permite asegurar durante todo el ejercicio económico un grado de ocupación elevado. Supone, a lo largo de todo el ciclo económico anual, cubrir los costes fijos y lograr un beneficio empresarial.

El crecimiento del sector de servicios residenciales para mayores está liderado por el sector privado lucrativo (empresas mercantiles), el cual ve la inversión en este sector como un "nicho de negocio" que atrae a grandes capitales y fondos de inversión, lo que se materializa en compras de establecimientos en funcionamiento y/o construcción de nuevos edificios bajo iniciativa propia, o a través de una "concesión" adjudicada por un poder público para la construcción de un establecimiento, ligada, generalmente, con la concesión de explotación del servicio. Se observa que la inversión privada en dichos centros se va concentrando en unas determinadas firmas configurando progresivamente un sector dominado por grandes holdings empresariales.

Según el informe Proyectos geriátricos en España (Alimarket, 2019), casi la mitad de las plazas (48,3\%) son gestionadas por empresas privadas, incluyendo tanto las de centros de titularidad privada como las de centros de titularidad pública cedidas a través de concesiones administrativas. Según el mencionado informe, el crecimiento del sector está liderado por el sector privado. El $40 \%$ de los proyectos de creación de nuevas camas en 2019 -aproximadamente 25.137-, están en manos de los principales grupos empresariales y la explotación de dicho sector es un negocio, según el mencionado informe: "La industria geriátrica española está dominada por grandes grupos, por lo menos en lo que respecta a las empresas punteras del sector. El sector geriátrico sigue siendo una oportunidad de negocio para inversores, empresas patrimonialistas, inversores foráneos y para los principales grupos gestores de residencias". 
1.1. Provisión pública y externalización de la gestión de los servicios residenciales para mayores

La presión de la demanda social por el envejecimiento de la población y el aumento de las personas en situación de dependencia, la escasa estructura de establecimientos públicos en los primeros años de la democracia, la existencia de una red privada de oferta de servicios residenciales y la mayor facilidad de las organizaciones privadas para invertir en infraestructuras residenciales, junto con la menor agilidad de la Administración en la gestión de los servicios y las restricciones presupuestarias para invertir y ampliar la reducida red de establecimientos públicos existentes, así como la minusvaloración del concepto de "gestión pública versus gestión privada", han influido en el desarrollo de la contratación externa de los servicios públicos con organizaciones privadas.

La externalización de los servicios sociales de responsabilidad pública se enmarca en lo que se denomina "economía mixta del bienestar", en la cual coexisten el sector público y el sector privado, pero con roles diferenciados; se reserva la competencia de la financiación al sector público y la de gestión al sector privado, aunque a la Administración Pública, como órgano contratante del servicio y, a la vez, como poder público, le compete la obligación de controlar la ejecución del contrato e intervenir y sancionar en caso de que el organismo proveedor no cumpla con los requisitos establecidos en el contrato.

La externalización de los servicios del bienestar debe contextualizase en el ámbito de las ideas neoliberales que se introdujeron con fuerza en el sector productivo en los años ochenta del siglo XX y que influenciaron la economía de los servicios sociales. La extensión de dichas teorías ensalzando las virtudes del sector privado y las deficiencias del sector público empujaron a una redefinición del rol del Estado de Bienestar. El Estado debía reducir su protagonismo en la provisión de la protección social mientras que el sector no-estatal (instituciones privadas lucrativas y no lucrativas) debía jugar un papel más importante en la oferta de servicios del bienestar. Esta concepción dio paso a lo que se llama economía mixta de bienestar o pluralismo del bienestar (welfare pluralism).

Algunos teóricos critican la irrupción del neoliberalismo en los servicios sociales señalando que al mismo tiempo que el pluralismo del bienestar ofrece una mayor y más variada oferta de servicios, también comporta un aumento de la desigualdad (restricciones en el acceso a servicios, selección adversa de los individuos, según complejidad de la discapacidad/ enfermedad, entre otros. El cambio del concepto de bienestar "centrado en el Estado" hacia el "pluralismo del bienestar" conduce a la privatización en la provisión de servicios reduciendo el rol del Gobierno a "proveedor del bienestar residual" (Mishra, 1995). Otros autores apuntan que la elección entre gestión directa y gestión indirecta de servicios de responsabilidad pública no se hace tanto basada en un estudio riguroso sobre qué tipo de gestión es la más eficiente, sino en otro tipo de circunstancias, como puede ser la existencia de un reducido número de proveedores, o la influencia política de determinadas instituciones que empujan a un determinado tipo de elección (Kramer, 1987).

En España, la economía mixta del bienestar en el ámbito de los servicios sociales se extiende a partir de la democratización de los ayuntamientos ${ }^{4} \mathrm{y}$ toma forma cuando el modelo de servicios sociales tiende hacia la universalización de las prestaciones, en un intento de equipararlo al resto de ámbitos del bienestar social (salud y educación), pero con su propia especificidad de ir configurándose cada vez más claramente como un modelo de provisión mixta, donde las entidades privadas (tanto lucrativas como no lucrativas) siguen jugando un papel relevante (Rodríguez Cabrero, 2004).

La eficiencia económica es el principal argumento que sostienen los teóricos del neoliberalismo en el ámbito de la economía de los servicios del bienestar, pero el debate entre eficiencia económica y eficacia social, entre gestión pública y gestión privada, sigue abierto sin que haya, por el momento, suficiente evidencia empírica que justifique la mejor alternativa.

La descentralización de las competencias de servicios sociales en las comunidades autónomas y el desarrollo de las leyes de servicios sociales favorecieron la expansión de instrumentos de colaboración público-privada, reduciendo a mínimos la gestión directa de los servicios sociales. Actualmente, la externalización de los servicios de responsabilidad pública está ampliamente extendida en todos los niveles de la Administración Pública -local, regional y estatal-. Incluso, una de las leyes más relevante en el ámbito social, la Ley 39/2006, de Promoción de la Autonomía Personal y Atención a las Personas en Situación de Dependencia (LAPAD), reconoce e impulsa la economía mixta del bienestar cuando en el artículo 6 configura una red de utilización pública que integra, de forma coordinada, centros y servicios, públicos y privados.

El progresivo aumento de la externalización de la gestión de servicios sociales no significa que no continúe existiendo la gestión directa en organizaciones públicas -especialmente en centros de la Administración local-, aunque actualmente suponga un porcentaje residual del total de plazas, tal y como se expone en el siguiente apartado. Una breve clasificación de los tipos de gestión es: gestión directa, cuando esta es realizada con medios y recursos de la propia Administración, aunque, en ocasiones, el financiador puede delegar la gestión en otros entes públicos (organismos autónomos administrativos, entidades mercantiles públicas, consorcios y fundaciones, entre otras) y gestión indirecta cuando esta es realizada a través de

\footnotetext{
${ }^{4}$ Primeras elecciones municipales en 1979.
} 
organizaciones privadas lucrativas o no lucrativas (sociedades mercantiles, asociaciones, fundaciones y cooperativas, entre otras).

\section{La calidad asistencial de las residencias para mayores desde la perspectiva del empleo}

En las residencias de mayores, el principal factor de producción son los "empleados" que atienden a las personas usuarias. La calidad de la atención dependerá, en gran parte, de las condiciones de trabajo de su personal. Durante la crisis sanitaria de la covid-19 se han evidenciado las dificultades de atención en las residencias por falta de suficientes cuidadores y de profesionales cualificados, y también se ha hecho evidente la precariedad de las condiciones laborales de dicho personal.

El objetivo de este apartado es describir la calidad del empleo en el conjunto de las organizaciones proveedoras de servicios residenciales para mayores, analizando los tipos de contratos de sus empleados y las retribuciones salariales a partir de los datos de la Muestra Continua de Vidas Laborales (MCVL) y de las bases de cotización de la Seguridad Social. Es un enfoque integral del empleo desde la perspectiva de las organizaciones que gestionan los servicios con independencia de si son plazas públicas o plazas privadas. En el Anexo se ofrece información de la base de datos MCVL utilizada para la realización de este estudio.

\subsection{Número de empleados: empleo total y empleo efectivo}

En el sector de los servicios residenciales para mayores, el elevado número de contratos de duración determinada (temporales), con pocos días o pocas horas de trabajo, junto con los contratos indefinidos a tiempo parcial, muestra la incorrección de utilizar el indicador de número total de empleados para describir la "fuerza de trabajo" realmente empleada en un centro. Por ello, se ha recurrido al concepto de "empleo efectivo", el cual mide el "personal equivalente a tiempo completo" de acuerdo con las horas anuales establecidas en el VII convenio colectivo marco estatal de servicios de atención a las personas dependientes. Para ello se tienen en cuenta tanto el número de días con contrato como el número de horas contratadas de cada uno de los trabajadores. La diferencia entre el "número total de empleados" y el "número total de empleo efectivo" es significativa. Aproximadamente, el número total de empleados al año se reduce en casi la mitad (42\%) al transformarse en "empleo efectivo". En 2018, el número de empleados es de 153.625, mientras que el “empleo efectivo" es de 89.248.

El sector ha experimentado un crecimiento considerable entre 2012 y 2018 , aumentando el número de empleos efectivos en $26.554,72$, lo que supone un incremento de más de un cuarenta por ciento (42,36\%) distribuyéndose en 9.623,73 empleos entre 2012-2015, y 16.930,99 empleos entre 2015-2018. Los mejores valores del segundo intervalo pueden deberse a la recuperación económica después del largo periodo de crisis iniciada en 2008, en el que tanto las familias como las Administraciones Públicas disponen de mayores recursos económicos para contratar plazas en residencias para mayores.

El Cuadro 2 muestra las tres dimensiones del empleo: el número total de empleos en el año, el número de empleos a final del ejercicio y el empleo efectivo durante el año. Asimismo, muestra el porcentaje de mujeres empleadas cuyas cifras son similares tanto desde la perspectiva de empleo efectivo como desde

Cuadro 2. Número de empleos y empleos efectivos (España)

\begin{tabular}{|c|c|c|c|c|c|c|}
\hline & & $\begin{array}{c}\text { № total empleados } \\
\text { en el año }\end{array}$ & $\begin{array}{l}\text { № total empleados } \\
\text { final año }\end{array}$ & $\begin{array}{c}\text { Porcentaje } \\
\text { empleados final año }\end{array}$ & $\begin{array}{l}\text { Empleo efectivo en } \\
\text { el año }\end{array}$ & $\begin{array}{c}\text { Porcentaje empleo } \\
\text { efectivo }\end{array}$ \\
\hline \multirow{3}{*}{2012} & Hombres & 11.775 & 7.725 & 11,0 & $6.803,74$ & 10,9 \\
\hline & Mujeres & 85.725 & 62.775 & 89,0 & $55.889,31$ & 89,1 \\
\hline & TOTAL 2012 & 97.500 & 70.500 & & $62.693,05$ & \\
\hline \multirow{3}{*}{2015} & Hombres & 15.625 & 10.225 & 12,2 & $8.468,48$ & 11, \\
\hline & Mujeres & 103.500 & 73.475 & 87,8 & $63.848,29$ & 88,3 \\
\hline & TOTAL 2015 & 119.125 & 83.700 & & $72.316,78$ & \\
\hline \multirow{3}{*}{2018} & Hombres & 21.275 & 13.550 & 13,2 & $11.257,21$ & 12,6 \\
\hline & Mujeres & 132.350 & 89.325 & 86,8 & $77 \cdot 990,56$ & 87,4 \\
\hline & TOTAL 2018 & 153.625 & 102.875 & & $89.247,77$ & \\
\hline
\end{tabular}

Fuente: Elaboración propia a partir de las MCVL. 
la situación a final del año, poniendo de relieve el elevado grado de feminización en estos centros.

\subsubsection{Características generales del empleo}

Los establecimientos residenciales para mayores se caracterizan por un elevado grado de feminización de los puestos de trabajo. Casi el $90 \%$ de los trabajadores son mujeres, aunque su peso sobre el total del empleo muestra una "cierta" tendencia decreciente, del 89,10\% en 2012 al 87,40\% en 2018. El número de empleo efectivo femenino ha aumentado en 22.101 entre 2012 y 2018 , lo que supone un incremento de casi el $40 \%(39,54 \%)$.

La proporción de mujeres en los diferentes tipos de organizaciones, que se analiza globalmente en el siguiente apartado, es bastante similar, entre un $86 \%$ y un $87 \%$. Con una proporción mayor figuran las corporaciones locales, con el 90,7\%, y las Instituciones religiosas, con el 89,7\%.

La edad media del conjunto de empleados es de 41 años y se ha venido manteniendo casi constante a lo largo del periodo. Casi el $60 \%$ de los trabajadores se sitúan en la franja entre 35 y 55 años. Algo menos del $60 \%$ de los trabajadores tienen un nivel de estudios primarios incluyendo algún grado de formación profesional $\left(E G B+F P_{1}\right)$ y el resto se distribuye entre un $20,4 \%$ con estudios secundarios (BUP + FP2) y un $22,6 \%$ con estudios superiores.

\subsection{Organizaciones gestoras de servicios residenciales para mayores}

Las organizaciones gestoras de servicios se identifican según la forma jurídica del empleador y las agrupamos en: organizaciones privadas con fin de lucro - sociedades anónimas, sociedades limitadas y uniones temporales de empresas (UTE) - , organizaciones privadas sin fin de lucro -fundaciones, asociaciones y cooperativas 5 -, organismos públicos - corporaciones locales y otros organismos públicos-, instituciones religiosas y, por último, el grupo "otros" agrupa aquellas empresas no encuadradas en ninguno de los apartados anteriores y, también, aquellas situaciones de trabajadores que a lo largo del año hayan cambiado de empresa encuadradas en diferentes grupos. El Gráfico 1 muestra la evolución de los empleos efectivos anuales por tipo de organización "gestora" sobre el total de empleos efectivos expresada en porcentajes.

El Gráfico 1 muestra cómo el conjunto de las organizaciones privadas con fin lucrativo gestionan casi un $70 \%$ de los empleados (empleos efectivos) del sector. La evolución muestra que dichas organizaciones mantienen la misma posición que en 2012. Se observa que en 2015 hay un incremento que no se mantiene en 2018. Una de las posibles explicaciones es que los trabajos de concesión de obra y gestión de servicios realizados por UTE finalizaran las obras y revirtieran a la Administración

Gráfico 1. Evolución de empleos efectivos por tipo de organización gestora de los servicios (España, \%)

Organizaciones privadas con fin de lucro

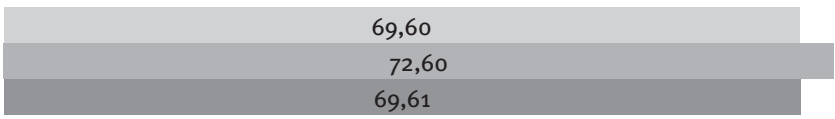

Organizaciones privadas sin fin de lucro
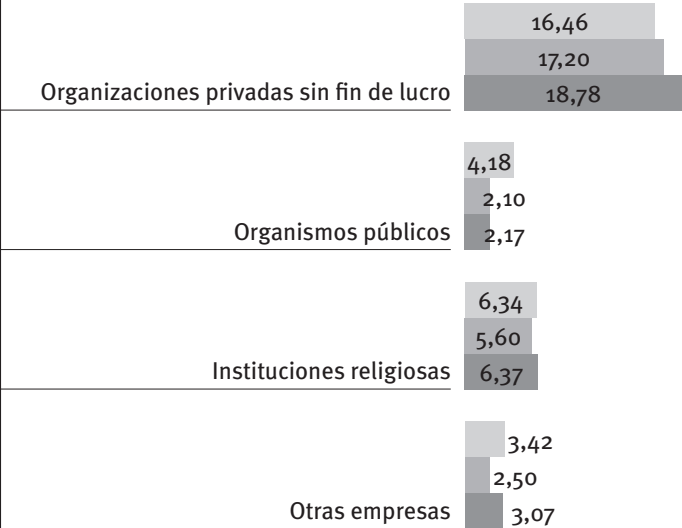
tanto las obras como la gestión de los servicios. Ello tiene sentido si se observa que el porcentaje de empleados gestionados por los organismos públicos ha aumentado en 2018.

Las organizaciones privadas no lucrativas (Tercer Sector) ocupan la segunda posición del ranking, con la gestión del 16,4\% del total de empleos efectivos del sector residencial para mayores. Se encuentran muy alejadas de la posición ocupada por las organizaciones privadas lucrativas. Muestran una evolución decreciente respecto al año 2012, perdiendo más de dos puntos porcentuales $(2,32)$ en dicho periodo. Ello induce a pensar que las nuevas formas de concertación con la Administración Pública para gestionar servicios personales de bienestar introducidas en la reforma de la Ley de Contratos del Sector Público en 2017 todavía no han dado sus frutos. Sin embargo, hay muestras de que este sector está en fase expansiva habilitando nuevos centros y ampliando el número de plazas en residencias para mayores tal como se puede apreciar en el siguiente texto.

La presencia del Tercer Sector, a pesar de su reducido tamaño en comparación con el grupo de "organizaciones privadas con ánimo de lucro", está en una senda expansiva. En septiembre de 2020 , la presencia del Tercer Sector supera las 91.000 camas, con más de 1.220 centros abiertos manteniendo, e incluso incrementando ligeramente su peso de años anteriores en el total de geriátricos de nuestro país. (Informe Alimarket/Sanidad, 2020)

Las organizaciones públicas, con un 4,2\% de empleos gestionados directamente, ocupan una posición residual en la lista. Este resultado contrasta con el hecho de que casi la mitad del total de usuarios de los servicios residenciales para mayores son financiados públicamente y que organismos de la Administración Pública son titulares de 5.457 centros residenciales para mayores (Imserso, 2019). Estos datos refuerzan la idea del elevado porcentaje de servicios públicos contratados externamente con organizaciones privadas.

Las instituciones religiosas, con el 6,3\% de los empleos gestionados directamente, contrastan con el hecho de que son titulares del $39,77 \%$ de las residencias del Tercer Sector y de aproximadamente 36.315 plazas, un $43,5 \%$ del total del sector no lucrativo (Alimarket, 2020), lo cual indica que contratan externamente la gestión de la mayoría de sus centros.

\subsection{La calidad de los contratos laborales}

En 2018, una cuarta parte del empleo efectivo ha sido realizado por trabajadores con contratos de duración determinada y se observa una evolución creciente a lo largo del periodo 2012-2018, pasando del $23,3 \%$ al $24,9 \%$. La proporción de este tipo de contratos es algo inferior en las mujeres que en los hombres, $24,8 \%$ frente al $25,5 \%$. Se observa también un aumento de trabajadores que han estado en ambos tipos de contrato a lo largo del año, del $2 \%$ en 2012 a casi el $5 \%$ en 2018 . Ello significa mayor movilidad entre contratos, de temporal a indefinido, o viceversa. La evolución del porcentaje de los contratos de duración determinada ha ido aumentando a lo largo de los años, pudiéndose apreciar un incremento de 1,6 puntos porcentuales entre 2012 y 2018 (Cuadro 3).

La proporción de contratos indefinidos, alrededor del $70 \%$, dista mucho de la cifra exigida en el VII Convenio colectivo marco estatal de servicios de atención a las personas dependientes ${ }^{6}$, el cual determina que los centros han de tener como mínimo un $80 \%$ de personal con contratos indefinidos. El porcentaje de contratos indefinidos muestra una tendencia decreciente durante el periodo, pasando del $74,7 \%$ en 2012 al 70,2\% en 2018 , y dando paso, por tanto, a un mayor número de contratos temporales.

Cuadro 3. Evolución del empleo efectivo por tipos de contrato (España)

\begin{tabular}{|c|c|c|c|c|c|c|c|c|c|}
\hline & \multicolumn{3}{|c|}{2012} & \multicolumn{3}{|c|}{2015} & \multicolumn{3}{|c|}{2018} \\
\hline & $\begin{array}{c}\text { Empleo } \\
\text { efectivo } \\
\text { (\%) }\end{array}$ & $\begin{array}{c}\text { Empleo } \\
\text { efectivo } \\
\text { hombres } \\
\text { (\%) }\end{array}$ & $\begin{array}{c}\text { Empleo } \\
\text { efectivo } \\
\text { mujeres } \\
(\%)\end{array}$ & $\begin{array}{l}\text { Empleo } \\
\text { efectivo } \\
\text { (\%) }\end{array}$ & $\begin{array}{c}\text { Empleo } \\
\text { efectivo } \\
\text { hombres } \\
\text { (\%) }\end{array}$ & $\begin{array}{c}\text { Empleo } \\
\text { efectivo } \\
\text { mujeres } \\
(\%)\end{array}$ & $\begin{array}{c}\text { Empleo } \\
\text { efectivo } \\
(\%)\end{array}$ & $\begin{array}{c}\text { Empleo } \\
\text { efectivo } \\
\text { hombres } \\
\text { (\%) }\end{array}$ & $\begin{array}{c}\text { Empleo } \\
\text { efectivo } \\
\text { mujeres } \\
\text { (\%) }\end{array}$ \\
\hline Contratos indefinidos & 74,7 & 72,1 & 75,0 & 73,3 & 70,5 & 73,6 & 70,2 & 68,6 & 70,4 \\
\hline $\begin{array}{l}\text { Contratos duración } \\
\text { determinada }\end{array}$ & 23,3 & 26,1 & 22,9 & 23,9 & 26,0 & 23,6 & 24,9 & 25,5 & 24,8 \\
\hline $\begin{array}{l}\text { Contratos varios } \\
\text { tipos }\end{array}$ & 2,0 & 1,8 & 2,1 & 2,8 & 3,5 & 2,7 & 4,9 & 5,9 & 4,8 \\
\hline Total & 100,0 & 10,85 & 89,15 & 100,0 & 11,71 & 88,29 & 100,0 & 12,61 & 87,39 \\
\hline
\end{tabular}

Fuente: Elaboración propia a partir de las MCVL de los años 2012, 2015 y 2018. 
Cuadro 4. Empleo efectivo según dedicación de jornada (España)

\begin{tabular}{|c|c|c|c|c|c|c|c|c|c|}
\hline & \multicolumn{3}{|c|}{2012} & \multicolumn{3}{|c|}{2015} & \multicolumn{3}{|c|}{2018} \\
\hline & $\begin{array}{c}\text { Empleo } \\
\text { efectivo } \\
(\%)\end{array}$ & $\begin{array}{c}\text { Empleo } \\
\text { efectivo } \\
\text { hombres } \\
\text { (\%) }\end{array}$ & $\begin{array}{c}\text { Empleo } \\
\text { efectivo } \\
\text { mujeres } \\
(\%)\end{array}$ & $\begin{array}{l}\text { Empleo } \\
\text { efectivo } \\
(\%)\end{array}$ & $\begin{array}{c}\text { Empleo } \\
\text { efectivo } \\
\text { hombres } \\
(\%)\end{array}$ & $\begin{array}{c}\text { Empleo } \\
\text { efectivo } \\
\text { mujeres } \\
(\%)\end{array}$ & $\begin{array}{l}\text { Empleo } \\
\text { efectivo } \\
\text { (\%) }\end{array}$ & $\begin{array}{c}\text { Empleo } \\
\text { efectivo } \\
\text { hombres } \\
\text { (\%) }\end{array}$ & $\begin{array}{c}\text { Empleo } \\
\text { efectivo } \\
\text { mujeres } \\
(\%)\end{array}$ \\
\hline Jornada completa & 78,5 & 76,9 & 78,6 & 75,61 & 74,84 & 75,71 & 75,1 & 71,1 & 75,7 \\
\hline Jornada parcial & 17,3 & 17,8 & 17,2 & 19,24 & 19,42 & 19,22 & 18,7 & 22,0 & 18,2 \\
\hline $\begin{array}{l}\text { Varios tipos de } \\
\text { jornada }\end{array}$ & 4,3 & 5,3 & 4,2 & 5,15 & 5,75 & 5,07 & 6,2 & 6,9 & 6,1 \\
\hline Total & 100,00 & 10,85 & 89,15 & 100,00 & 11,71 & 88,29 & 100,00 & 12,61 & 87,39 \\
\hline
\end{tabular}

Fuente: Elaboración propia a partir de las MCVL.

Cuadro 5. Detalle de los principales contratos temporales (España)

\begin{tabular}{|c|c|c|c|}
\hline \multicolumn{4}{|c|}{ Empleos efectivos por tipo contrato temporal, en porcentaje $\left(^{\star}\right)$} \\
\hline & 2012 & 2015 & 2018 \\
\hline Obra o servicio & 20,38 & 16,72 & 13,69 \\
\hline Eventual & 39,94 & 40,42 & 41,06 \\
\hline Interinidad & 35,80 & 37,44 & 39,57 \\
\hline Empleos grupo/total contratos temporales & 96,10 & 94,58 & 94,31 \\
\hline \multicolumn{4}{|c|}{ Otros indicadores } \\
\hline Media días contrato año "grupo" & 127,46 & 116,1 & 112,72 \\
\hline Media contratos "grupo" & 2,44 & 2,46 & 2,38 \\
\hline
\end{tabular}

(*) Los porcentajes se miden sobre el total de empleos efectivos con contrato temporal. Fuente: Elaboración propia.

\subsection{Empleo efectivo según dedicación jornada}

En 2018, casi el $19 \%$ de los empleos efectivos son de jornada parcial, habiendo experimentado un ligero incremento entre 2012 y 2018 , del $17,3 \%$ al $18,7 \%$. Es algo mayor la proporción de hombres con jornada parcial que la proporción en las mujeres ( $22 \%$ versus $18,2 \%$ en 2018), diferencia que se ha visto acentuada a lo largo del periodo analizado. Ello se debe a la mayor contratación de hombres que de mujeres en contratos temporales.

En correspondencia, casi el $76 \%$ de las mujeres tienen jornada completa, mientras que en los hombres es el $71 \%$. Asimismo, hay un $6,2 \%$ de empleados que han pasado de un tipo de jornada a otra, proporción que ha ido aumentando a lo largo del periodo (Cuadro 4).

\subsection{Análisis de los contratos temporales en los servicios residenciales para mayores}

Una de las principales características laborales es la elevada temporalidad del empleo y las características de los contratos temporales. En el Cuadro 5 se puede observar que casi un $95 \%(94,31 \%)$ del total de contratos temporales se agrupan en tres tipos: "obra o servicio", "eventual” e "interinidad”, formando un grupo que denominamos "grupo soporte de la plantilla fija de la empresa”. Los contratos eventuales y los contratos de interinidad 7 son los predominantes en el "grupo soporte", distribuyéndose en 2018 casi en la misma proporción $-41 \%$ para los eventuales y $40 \%$ para los de "interinidad"-. El resto de contratos temporales incluye contratos de formación, prácticas, inserción, persona con discapacidad y relevo, entre otros.

La elevada proporción de contratos de interinidad y su continuo crecimiento lleva a reflexionar sobre las condiciones laborales de los trabajadores en plantilla de las empresas. Se entrevé una elevada tasa de "sustituciones" por bajas temporales de enfermedad. Asimismo, la elevada proporción de contratos de “obra y servicio" y contratos "eventuales” induce a pensar que la función principal de este personal es la de "complementar" las necesidades de personal de plantilla de los centros. El resultado final es que el porcentaje de contratos temporales incluidos en lo que se ha denominado "grupo soporte" apenas ha variado a lo largo del periodo analizado.

Es significativo que el indicador "tasa de estabilidad" definido en el Convenio marco estatal de servicios a las personas dependientes se construya excluyendo del cómputo los contratos de interinidad, además de los de prácticas y formación y aprendizaje. Así,

7 En los contratos de interinidad se excluyen los de carácter administrativo por ser residuales. 
siguiendo dicho criterio resulta que, como media, el sector cumple el porcentaje de estabilidad fijado en un $80 \%$ en el mencionado convenio.

Otros indicadores a considerar de los contratos temporales incluidos en el grupo "soporte" son el elevado número de días de contrato por individuo y año, que alcanza una media de más 100 días, lo que equivale, aproximadamente, a casi cuatro meses. Además, el número de contratos por individuo y año es de 2,38 en 2018. Se observa, además, que un mismo empleado puede alargar los días trabajados al año enlazando diferentes modalidades de contrato temporal definidos en el grupo soporte.

Entre otras características de los contratos temporales, se destaca que la retribución salarial suele ser la mínima establecida por el convenio, no tienen derecho a pluses de antigüedad, ni horas de formación y que, por ejemplo, el contrato de interinidad no tiene derecho a indemnización cuando finaliza el contrato.

El conjunto de indicadores señalados en este apartado da idea de la elevada precariedad laboral de los empleos temporales en los servicios residenciales para mayores.

\subsubsection{Tasa de temporalidad de los contratos}

La tasa de temporalidad mide la proporción de contratos temporales sobre el total de contratos en el año. España se destaca por la elevada tasa de temporalidad laboral que duplica la tasa media de la EU 28, pero la tasa de temporalidad en los establecimientos residenciales para mayores triplica a la de España y es seis veces mayor que la de la media de la Unión Europea, mostrando la elevada precariedad laboral de este sector. Se observa, además, que dicha tasa de temporalidad ha aumentado considerablemente ( 4,6 puntos porcentuales) en el periodo 2012-2018 (Cuadro 6).

Cuadro 6. Tasa de temporalidad

\begin{tabular}{|l|c|c|c|}
\cline { 2 - 4 } \multicolumn{1}{c|}{} & $\mathbf{2 0 1 2}$ & $\mathbf{2 0 1 5}$ & $\mathbf{2 0 1 8}$ \\
\hline EU 28 & 10,7 & 11,1 & 11,2 \\
\hline España & 19,2 & 20,7 & 21,9 \\
\hline Residencias mayores & 62,4 & 65,8 & 67,0 \\
\hline
\end{tabular}

Fuente: Elaboración propia con datos MCVL y Eurostat (tesem_110). en 2018 y, también, es un $24 \%$ inferior al salario bruto anual del sector servicios, que según el INE ${ }^{8}$ es de 23.325,64 euros para dicho año. Esta importante diferencia salarial es una muestra significativa de la precariedad laboral de los trabajadores en los servicios residenciales para mayores. A continuación, se realiza una exposición detalla de las retribuciones salariales desde diferentes perspectivas del empleo.

\subsection{Los salarios desde la perspectiva de los tipos de contratos}

El desglose del salario hora por tipo de contrato pone de manifiesto que no cobran igual los que tienen contrato indefinido que aquellos con contrato de duración determinada. En general, los asalariados con contrato de duración determinada perciben un $4,2 \%$ menos que los que tienen un contrato indefinido. La diferencia es más pronunciada en los hombres que en las mujeres $13,3 \%$ en hombres y $2,7 \%$ en mujeres). Asimismo, la brecha salarial es más pronunciada en los contratos indefinidos $(12,1 \%)$ que en los contratos temporales $(1,3 \%)$, como se puede ver en el Cuadro 7.

Cuadro 7. Salario hora y brecha de género por tipo de contrato (España, año 2018)

\begin{tabular}{|l|c|c|c|}
\cline { 2 - 4 } & \multicolumn{3}{c|}{ Euros hora } \\
\cline { 2 - 4 } & 2012 & 2015 & 2018 \\
\hline Hombres & 10,21 & 10,31 & 10,55 \\
\hline Mujeres & 9,05 & 9,36 & 9,84 \\
\hline Brecha género & $\mathbf{- 1 1 , 4} \%$ & 9,48 & 9,94 \\
\hline
\end{tabular}

Fuente: Elaboración propia a partir de las MCVL.

El salario hora', en el periodo 2012-2018 ha aumentado un $8,2 \%$, incremento superior a la variación del IPC acumulado durante el mismo periodo de tiempo (3,21\%). Ello muestra un esfuerzo de las organizaciones para acercar salarios a los establecidos en el convenio laboral. La brecha de género muestra una progresiva disminución de 4,7 puntos porcentuales entre 2012, y 2018, aunque en el 2018 todavía las mujeres ganan en promedio un $6,7 \%$ menos que los hombres.

\section{Los salarios de los empleados en los establecimientos residenciales para mayores}

La retribución salarial media anual en los servicios residenciales para mayores es de 17.813,31 euros en 2018. Dicho importe es inferior al salario bruto anual de 24.009,12 euros para el conjunto de la economía
${ }^{8}$ Encuesta de estructura salarial, INE, 2018.

9 Se toma como unidad básica salarial el "salario hora", teniendo en cuenta las bases de las cotizaciones anuales de los trabajadores en el CNAE 873 y tomando como referencia 1.792 horas anuales fijadas en el VII Convenio colectivo de servicios de atención a las personas dependientes y desarrollo de la promoción de la autonomía personal, aplicable desde 2015 y que a efectos este estudio también se aplica en el 2012. 


\subsection{Los salarios desde la perspectiva de las categorías profesionales}

Uno de los factores que muestra la baja calidad del empleo en los centros residenciales de mayores es la gran concentración de trabajadores en las categorías profesionales bajas. Se analizan los contratos desde la perspectiva de las categorías profesionales, identificadas en los grupos de cotización de la Seguridad Social, donde cada contrato se encuadra en un grupo determinado para su correspondiente cotización. Los grupos de cotización ${ }^{10}$ se determinan en función de categorías profesionales y a cada categoría se le asigna una cuota mensual, que puede variar en función del salario y el tipo de trabajo que se desempeña. En este artículo se han agrupado las categorías profesionales en tres niveles: categoría alta, media y baja. Así, la categoría alta incluye los grupos de cotización 1 y 2, la categoría media incluye los grupos 3, 4 y 5 y la categoría baja, el resto de los grupos. Estas categorías son indicativas de las características del puesto que ocupan y de la retribución salarial que les corresponde, más que de la cualificación profesional de los trabajadores.

Los resultados muestran que, de promedio, el $80 \%$ de los empleos están encuadrados en las categorías profesionales más bajas. Ello da idea del nivel de titulaciones exigidas al personal para trabajar en dichos centros y de la escasa proporción de trabajadores con niveles profesionales, reconocidos, de grado medio o superior. El resto de los trabajadores se distribuyen del siguiente modo: un $13 \%$ en el grupo de categoría media -titulados de grado medio y cargos de responsabilidad mediay un $7 \%$ en el grupo de categoría alta -titulados superiores, directores y altos cargos-.
Se desglosa el salario entre bruto y neto para proporcionar una "foto" más precisa del alcance de las retribuciones salariales de los empleados en los servicios residenciales para mayores. El salario neto se obtiene aplicando una retención del $20 \%$, que incluye la parte correspondiente de la cotización a la Seguridad Social y la retención del IRPF ${ }^{11}$. Cabe precisar que los porcentajes de retención utilizados como hipótesis pueden variar significativamente si cambia la normativa que los regula.

Los resultados obtenidos muestran que un $80 \%$ de los empleados perciben, como media, un salario neto mensual inferior a mil euros, no mostrando diferencias significativas entre hombres y mujeres en la categoría laboral "baja”. En cambio, se perciben diferencias más elevadas en la categoría alta y, probablemente superior a la reflejada en dicho cuadro teniendo en cuenta los “topes” de cotización en los salarios más elevados.

La comparación salarial entre hombres y mujeres dentro de la misma categoría profesional pone de relieve que la brecha salarial de los empleados encuadrados en la categoría baja es muy pequeña $(1,5 \%)$. Se intuye que cuando los salarios son bajos no hay mucho margen para disminuirlos aún más, mientras que en las categorías media y alta la brecha de género aumenta, alcanzando cifras bastantes similares, $7,5 \%$ y $7,1 \%$, respectivamente (Cuadro 8 ).

La media de las retribuciones salariales está dentro de lo estipulado en el convenio del sector. Sin embargo, está muy por debajo del salario medio del "sector servicios" tal como se ha expuesto anteriormente.

Cuadro 8. Salario bruto y neto por categorías profesionales y género (España, año 2018)

\begin{tabular}{|c|c|c|c|c|c|c|c|c|}
\hline & & & & lario bruto $\mathrm{m}$ & & & lario neto $\mathrm{me}$ & \\
\hline & $\begin{array}{c}\text { Total } \\
\text { individuos } \\
\text { (\%) }\end{array}$ & $\begin{array}{c}\text { Brecha de } \\
\text { genero }\end{array}$ & $\begin{array}{c}\text { Media } \\
\text { euros/mes }\end{array}$ & $\begin{array}{c}\text { Hombres } \\
\text { euros/mes }\end{array}$ & $\begin{array}{c}\text { Mujeres } \\
\text { euros/mes }\end{array}$ & $\begin{array}{c}\text { Media } \\
\text { euros/mes }\end{array}$ & $\begin{array}{l}\text { Hombres } \\
\text { euros/mes }\end{array}$ & $\begin{array}{c}\text { Mujeres } \\
\text { euros/mes }\end{array}$ \\
\hline Categoria alta & 13,7 & $-7,1 \%$ & 1.725 & 1.828 & 1.699 & 1.380 & 1.462 & 1.359 \\
\hline Categoria media & 6,7 & $-7,5 \%$ & 1.388 & 1.484 & 1.373 & 1.110 & 1.187 & 1.098 \\
\hline Categoria baja & 79,6 & $-1,5 \%$ & 1.184 & 1.201 & 1.182 & 948 & 961 & 946 \\
\hline Total & & $-6,7 \%$ & 1.272 & 1.350 & 1.260 & & 1.018 & 1.008 \\
\hline
\end{tabular}

Salario año = salario mes por 14 pagas.

Fuente: Elaboración propia a partir de las MCVL.

${ }^{10}$ Grupo 1: ingenieros y licenciados. Grupo 2: ingenieros técnicos, peritos y ayudantes titulados. Grupo 3: jefes administrativos y de taller. Grupo 4: ayudantes no titulados. Grupo 5: oficiales administrativos. Grupo 6: subalternos. Grupo 7: auxiliares administrativos. Grupo 8: oficiales de primera y segunda. Grupo 9: oficiales de tercera y especialistas. Grupo 10: peones. Grupo 11: aquellos trabajadores menores de 18 años, sea cual sea su categoría profesional.
${ }^{11}$ Se estima un $4,7 \%$ de retención por la cotización a la Seguridad Social y un $15 \%$ como valor medio de retención del IRPF, teniendo en cuenta que esta varía en función de las circunstancias familiares y del importe de la remuneración anual estimada. 


\subsection{Retribuciones salariales por tipo de organización}

En este apartado se muestran los resultados de la media mensual de las retribuciones salariales por tipos de organización y por género. El resultado muestra que no hay diferencias significativas entre los grupos de organizaciones, excepto en el de “organismos públicos”, que muestra una diferencia salarial del $33 \%$ por encima de la media. Entre las posibles explicaciones de dicha diferencia se apuntan las siguientes: que algunos organismos públicos gestionen "centros de referencia” que requieran una ratio de personal más elevado, o que realmente los salarios pagados sean superiores a los de otras organizaciones. Asimismo, cabe recordar que las organizaciones públicas gestionan un reducido número de trabajadores con relación al total.

Respecto a la brecha de género, los organismos públicos destacan por ser los que la tienen más elevada, siendo superior a la media en 8,2 puntos. En segunda posición se sitúan las organizaciones religiosas con 4,2 puntos superior a la media. Las "organizaciones con fin lucrativo y el grupo de "otras organizaciones" son las que muestran una brecha inferior a la media (cuadro 9).

Cuadro 9. Salarios brutos por tipo de organización (España, año 2018)

\begin{tabular}{|l|c|c|c|c|}
\cline { 2 - 5 } \multicolumn{1}{c|}{} & $\begin{array}{c}\text { Media } \\
\text { (euros/ } \\
\text { mes) }\end{array}$ & $\begin{array}{c}\text { Hombres } \\
\text { (euros/ } \\
\text { mes) }\end{array}$ & $\begin{array}{c}\text { Mujeres } \\
\text { (euros/ } \\
\text { mes) }\end{array}$ & $\begin{array}{c}\text { Brecha } \\
\text { género }\end{array}$ \\
\hline $\begin{array}{l}\text { Organizaciones } \\
\text { lucrativas }\end{array}$ & 1.242 & 1.309 & 1.231 & $-5,9 \%$ \\
\hline $\begin{array}{l}\text { Organizaciones } \\
\text { no lucrativas }\end{array}$ & 1.326 & 1.422 & 1.310 & $-7,9 \%$ \\
\hline $\begin{array}{l}\text { Organizaciones } \\
\text { públicas }\end{array}$ & 1.689 & 1.947 & 1.657 & $-14,9 \%$ \\
\hline $\begin{array}{l}\text { Organizaciones } \\
\text { religiosas }\end{array}$ & 1.231 & 1.362 & 1.214 & $-10,9 \%$ \\
\hline Otras & 1.171 & 1.202 & 1.166 & $-3,0 \%$ \\
\hline Total & 1.272 & 1.350 & 1.260 & $-6,7 \%$ \\
\hline
\end{tabular}

Salario año = salario bruto mes por 14 pagas

Fuente: Elaboración propia a partir de las MCVL.

\section{El empleo y su incidencia en la gestión de situaciones de la covid-19 en residencias para mayores}

Es arriesgado ofrecer un balance de la afectación de la covid-19 en las residencias de mayores cuando en el momento de escribir este artículo (octubre de 2020) la crisis sanitaria todavía está en su apogeo. Algunas cifras aproximadas apuntan que, desde principios de marzo hasta el 27 de agosto, casi 20.000 personas mayores han perdido la vida a causa de la covid-19 y que alrededor de dos terceras partes de los fallecimientos relacionados con esta se habrían producido en establecimientos residenciales para mayores (CSIC, 2020). Sin embargo, estas cifras deben ser tomadas con cautela debido a sus potenciales sesgos y limitaciones en las informaciones facilitadas por las comunidades autónomas (Zalakain, Davey y Suárez-González, 2020).

La (des)coordinación intersectorial en muchos lugares del territorio nacional ha demostrado ser una de las claves explicativas de la magnitud alcanzada por esta crisis, tanto en el ámbito políticoadministrativo (Ministerio y Consejerías), como en el organizativo (residencias y centros sanitarios). En algunas CC.AA. se han producido problemas de coordinación sectorial que han retrasado la protección de las residencias, a pesar de ser los lugares donde se han concentrado buena parte de los problemas más acuciantes. (Del Pino et al., 2020)

Los escasos (débiles) controles ejercidos por la Administración para comprobar y exigir el cumplimiento de los requisitos establecidos en los contratos/conciertos para la gestión de plazas con financiación pública y, en general, de los requisitos exigidos para la acreditación de los centros, han puesto al descubierto deficiencias estructurales con repercusiones en la atención a las personas usuarias. Entre los principales problemas que han aflorado destacan los de insuficiencia de personal, así como la elevada precariedad laboral, la cual se ha expuesto a lo largo de este artículo. Las condiciones laborales y salariales existentes han dificultado encontrar personal dispuesto a realizar sustituciones en situaciones difíciles, dando lugar a plantillas de personal más infradimensionadas que las previamente existentes. Cabe recordar que, para suplir dicho efecto, algunas Administraciones optaron por permitir la contratación laboral aunque las personas solicitantes no dispusieran de la cualificación profesional pertinente.

La falta de recopilación de la información, o del adecuado tratamiento de esta, junto con una política de restricciones del gasto público para concertar plazas públicas, han incidido en una manifiesta relajación de la Administración en la exigencia a los centros en lo referente al cumplimiento de ciertas normas, destacando dos de ellas: el cumplimiento de la ratio de personal y la exigencia de unos determinados porcentajes de cualificaciones profesionales.

La ratio de personal/usuario fue definida a partir del Acuerdo del Consejo Territorial sobre criterios comunes de acreditación para garantizar la calidad de los centros y servicios del SAAD ${ }^{12}$ y publicado por resolución de 2 de diciembre de 2008 , siguiendo lo preceptuado en el artículo 35.1 de la LAPAD "se establecerán estándares de calidad para cada uno de los servicios, previo acuerdo del Consejo Territorial". El acuerdo determina una ratio global media para las residencias de mayores en situación de dependencia

\footnotetext{
${ }_{12}$ SAAD es el acrónimo del Sistema para la Autonomía y Atención a la Dependencia, mientras que SISSAD el de su sistema de informa-
} ción. 
exigible a partir de 2011, desglosando la ratio en los siguientes mínimos: un 0,45 para las personas con un grado 2 de dependencia y un 0,47 para las personas con un grado 3 de dependencia, los cuales deben incluir un mínimo de personal "cuidador/gerocultor" de 0,27 y 0,28 , respectivamente. La ratio se aplicará en cada centro con relación al número de usuarios valorados con el respectivo grado de dependencia.

El cumplimiento de la ratio de personal es uno de los requisitos exigidos para la acreditación de los centros, servicios y entidades privadas para su incorporación en la red pública de servicios del SAAD, pero también es exigible para aquellos otros centros que sean acreditados para acoger personas beneficiarias de las prestaciones vinculadas establecidas en la LAPAD.

En cuanto al cumplimiento de las ratios de personal en los establecimientos de servicios residenciales para mayores, el Imserso no ofrece ningún tipo de información, ni en el SISAAD, ni en cualquier otro documento. Para suplir dicha falta de información, se ha realizado una "estimación" ${ }^{13}$ a nivel de comunidades autónomas a través de la información de empleo efectivo obtenida a través de la MCVL y de la información del CSIC sobre plazas residenciales de 2018 , estimando una ocupación del $90 \%$. Los resultados muestran que la media de la ratio de personal por plaza ocupada de 0,27 está muy lejos de la establecida en el acuerdo del Consejo Territorial entre 0,45 y 0,47. Solo la Comunidad Valenciana, con 0,43 , se aproxima la mencionada ratio; otras comunidades, como La Rioja y Navarra se acercan a la ratio estándar (Gráfico 2).

Otra de las manifestaciones de la relajación de la Administración es el hecho de ir posponiendo la exigencia de unos determinados porcentajes de cualificaciones profesionales establecidos en el mencionado acuerdo. Dichos niveles debían alcanzarse a finales de 2015 , pero sucesivos acuerdos han ido prorrogando dicha exigencia, actualmente, hasta el 2022 (Resolución 11 de diciembre de 2017 de la Secretaría de Estado de Servicios Sociales e Igualdad). Ello da lugar a una insuficiencia de perfiles profesionales adecuados para la atención a las personas usuarias.

La escasez de personal sanitario en las residencias ha incidido en la manifiesta incapacidad para actuar de forma rápida y adecuada ante los síntomas de la

Gráfico 2. Estimación de la ratio "Empleo efectivo/plazas ocupadas" (España)

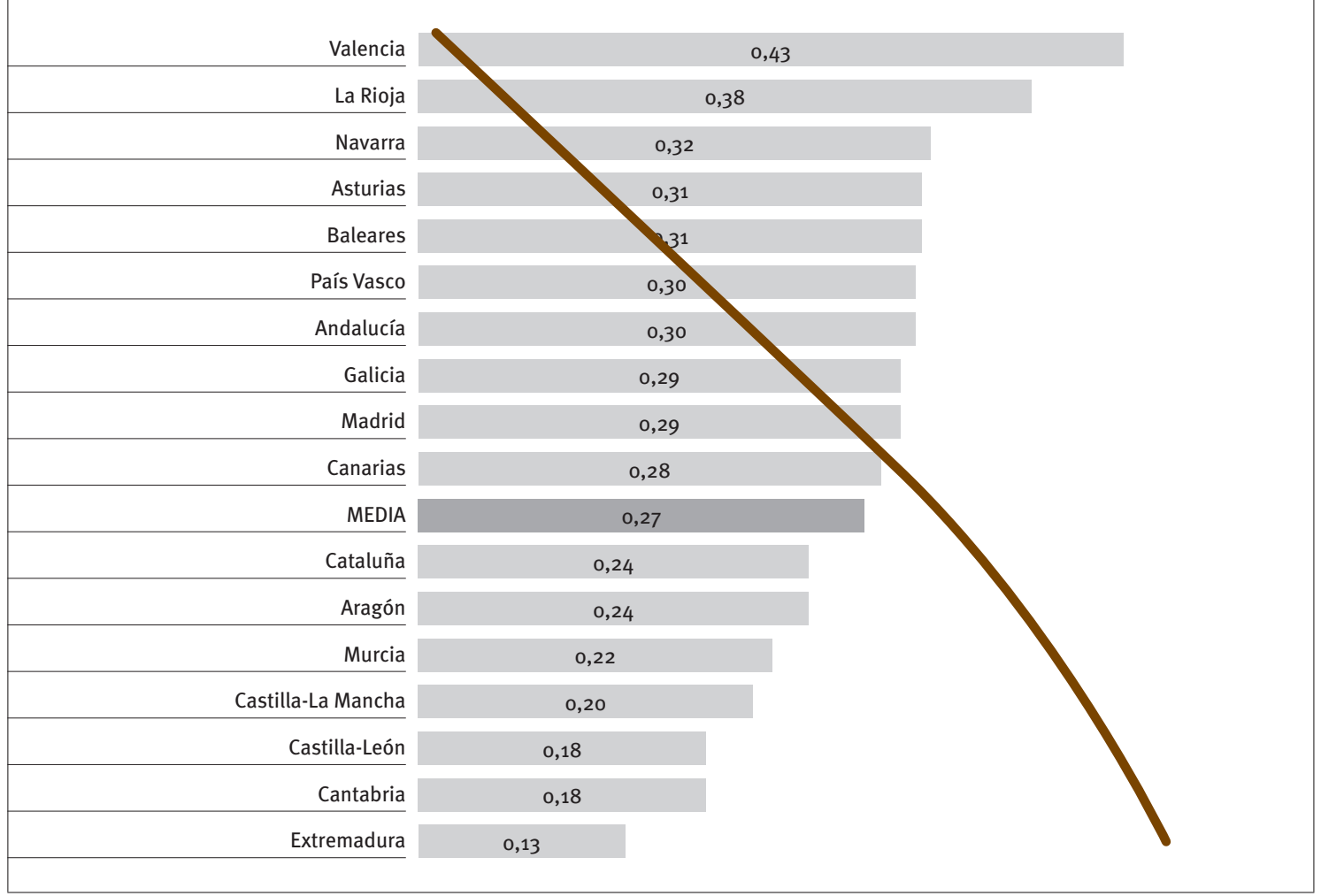

Fuente: Elaboración propia.

${ }_{13}$ Es una aproximación a la ratio personal/plaza, ya que se desconocen otros factores en qué proporción las plazas están ocupadas por usuarios de grado 2 y de grado 3 o en qué proporción hay usuarios que no están en situación de dependencia. 
enfermedad y/o el tratamiento durante el proceso, y ante las restricciones para acceder a los centros hospitalarios o a los centros de atención primaria. La mayoría de las residencias atienden a personas en situación de dependencia y una gran proporción de ellas tienen pluripatologías que requieren control y atención médica continuada más o menos intensa. En cambio, los centros no suelen disponer, ya que no siempre es exigible según las normativas de las comunidades autónomas para la acreditación de centros residenciales de personal de sanitario, ni siquiera con contratos de algunas horas de atención semanales. Solo algunas de ellas, las más grandes o las más lujosas, disponen de este tipo de personal de forma permanente.

La coordinación sociosanitaria es uno de los objetivos más “codiciados”, pero todavía irresueltos a nivel nacional. Cada autonomía establece sus propias vinculaciones entre los servicios sociales y los servicios sanitarios, pero el grado de éxito en esta crisis sanitaria no parece que haya sido excelente. El elevado grado de saturación de los centros de atención primaria, así como la selección adversa aplicada en algunos hospitales, ha incidido en que muchos de los residentes con covid-19 no tuvieran acceso a la atención sanitaria o al centro hospitalario en situaciones de gravedad extrema.

Una dotación insuficiente de personal social y sanitario repercute en una inadecuada calidad de atención a las personas usuarias. La dispersión normativa entre los diferentes niveles territoriales sobre centros residenciales y requisitos de autorización y acreditación, junto con un deficiente mecanismo de recopilación de datos, siguen dificultando el diseño de las estrategias más adecuadas para la atención residencial (Defensor del Pueblo, 2020).

\section{Conclusiones}

La crisis sanitaria de la covid-19 ha sido el revulsivo que ha aflorado numerosas deficiencias alrededor de la coordinación y gestión de las residencias para mayores. Se han producido problemas en el ámbito organizativo entre centros sociales y sanitarios que han afectado a la atención sanitaria de los residentes, pero sobre todo se ha puesto de manifiesto la precariedad laboral de los empleados y la insuficiente dotación de personal para atender a un colectivo muy vulnerable como es el de las personas mayores en situación de dependencia, así como los fallos de la Administración para controlar y garantizar la adecuada calidad de los servicios residenciales.

La precarización laboral y la insuficiente dotación de personal son dos de los hechos relevantes que han motivado situaciones de desatención extrema en el contexto de la crisis sanitaria. El convenio de personal que regula este sector establece unos salarios tan bajos que el resultado es una retribución anual bruta hasta un $24 \%$ inferior a la del conjunto del sector servicios, y todo ello en el marco de unas condiciones laborales agotadoras tanto física como mentalmente. La importante proporción de empleo con contratos de duración determinada y elevada tasa de temporalidad no son una garantía para ofrecer un servicio de calidad, ya que las empresas/organizaciones no pueden "fidelizar" a sus trabajadores; estos aprovechan cualquier oportunidad para moverse a otros lugares de trabajo mejor remunerados. Asimismo, las personas atendidas sufren emocionalmente los cambios incesantes de los trabajadores, no pudiendo establecer una relación continuada de confianza con la o las personas cuidadoras.

La Administración Pública ha fallado también en no establecer los mecanismos de control para asegurar el cumplimiento de los mínimos de dotación de personal -ratio personal/usuario- establecidos a través del acuerdo del Consejo Territorial para las residencias que atiendan a personas en situación de dependencia. La estimación de la ratio "empleo efectivo/plaza ocupada" para el conjunto de servicios residenciales, con un resultado de 0,27, está muy alejado del estándar mínimo fijado entre 0,45 y 0,47 acordado por el Consejo Territorial publicado en la Resolución de 2 de diciembre de 2008. Ello, sin lugar a dudas, tiene unas importantes consecuencias en la calidad asistencial.

Las técnicas utilizadas de concertación de plazas públicas a través de concursos públicos regulados por la Ley de Contratos del Sector Público (LCSP) han beneficiado a las organizaciones privadas lucrativas, excluyendo de facto a las organizaciones no lucrativas, las cuales no podían competir por las exigencias de solvencia económica y otros requisitos prácticamente inaccesibles para ellas. La reforma de la LCSP de 2017 abre la puerta a una nueva vía de colaboración entre las organizaciones privadas no lucrativas y las Administraciones Públicas. Se prevé que la diversificación y ampliación de los instrumentos de concertación junto con la incorporación de otros criterios, además del económico, en la adjudicación de las plazas repercuta en la mejora de la calidad asistencial de los centros.

La externalización de servicios públicos es una realidad que ha venido para quedarse, ya que se constata un escaso nivel de gestión directa de los servicios públicos residenciales para mayores. En cualquier caso, los criterios de eficiencia y eficacia deben ser los referentes para la financiación de las plazas públicas a las organizaciones proveedoras de servicios residenciales. Las Administraciones deberían reforzar el control del cumplimiento de los requisitos para la acreditación de los centros en general y de aquellos otros establecidos en las cláusulas de los conciertos/acreditaciones acordados con ellas. La implementación de controles realizados por organismos externos independientes mediante la exigencia de presentación de auditorías de cuentas anuales incluyendo la auditoria salarial y la vigilancia de las ratios personal/usuario constituiría 
un instrumento de ayuda para las Administraciones Públicas para verificar el cumplimiento de los requisitos de gestión exigidos a los centros.

Como conclusión final, cabe señalar como urgente la reparación de las deficiencias en la gestión y organización del empleo observadas en las residencias para mayores, así como la dignificación de las condiciones laborales del personal de atención directa a los usuarios. 


\section{Referencias bibliográficas}

ALIMARKET (2019): Informe 2019 de Proyectos Geriátricos en España, 〈https://www.alimarket.edad/ informe/293352>.

ALIMARKET/SANIDAD (2020): Informe Septiembre/Octubre, 2020, <https://www.alimarket.es/sanidad/ informe/319975/informe-2020-de-geriatricosdel-tercer-sector-en-espana/2/2188bb43d4bb5 8a07d2oeefb93049b71>.

CENTRO SUPERIOR DE INVESTIGACIONES CIENTÍFICAS (2020): "Envejecimiento en red". Estadística centros residenciales mayores 2019, 〈https:// www.envejecimiento.CSIC.es〉.

DEFENSOR DEL PUEBLO (2020): Atención a personas mayores. Centros residenciales. Separata del Informe anual 2019, Madrid, <https:// www.defensordelpueblo.es/wp-content/ uploads/2020/05/Separata_personas_ mayores_centros_residenciales.pdf .

DÍAZ DÍAZ, B. (2012): “Estimación del coste de la atención a la dependencia en centros residenciales y de atención diurna o nocturna", Zerbitzuan, ํㅜ 52 .

DEL PINO, E.; MORENO-FUENTES, F.J.; CRUZ-MARTÍNEZ, G.; HERNÁNDEZ-MORENO, J.; MORENO, L; PEREIRAPUGA, M.; Y PERNA, R. (2020): Informe Gestión Institucional y Organizativa de las Residenciad de Personas Mayores y COVID-19: Dificultades y aprendizajes, Instituto de Políticas y Bienes Públicos (IPP-CSIC), Madrid, 〈http://ipp. csic.es/sites/default/files/content/project/ mc-covid19_-296276/informe_residencias_ covid-19_ipp-csic.pdf .

GOBIERNO DE ESPAÑA (2008): Resolución de 2 de diciembre de 2008, de la Secretaría de Estado de Política Social, Familias y Atención a la Dependencia y a la Discapacidad, por la que se publica el Acuerdo del Consejo Territorial del Sistema para la Autonomía y Atención a la Dependencia, sobre criterios comunes de acreditación para garantizar la calidad de los centros y servicios del Sistema para la Autonomía y Atención a la Dependencia, 〈https://www.boe.es/eli/es/ $\mathrm{res} / 2008 / 12 / 02 /(2)>$.

- (2017): Resolución de 11 de diciembre de 2017, de la Secretaría de Estado de Servicios Sociales e Igualdad, por la que se publica el Acuerdo del Consejo Territorial de Servicios Sociales y del Sistema para la Autonomía y Atención a la Dependencia, que modifica parcialmente

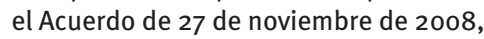
sobre criterios comunes de acreditación para garantizar la calidad de los centros y servicios del Sistema para la Autonomía y Atención a la Dependencia, 〈https://www.boe.es/eli/es/ $\mathrm{res} / 2017 / 12 / 11 /(5)\rangle$.

IMSERSO (2019): Informe servicios sociales dirigidos a personas mayores en España. Diciembre 2018, 〈https://www.imserso.es/imserso_01/ documentacion/estadisticas/ssppmm esp/2018/index.htm>.

KRAMER, R.M. (1987): "Nonprofit social service agencies. Research considerations", The Nonprofit Sector and the Modern Welfare State, Political Science Quarterly, Vol. 104, The Academy of Political Science.

MARBÁN GALLEGO, V.; MONTSERRAT CODORNIU, J. y RODRÍGUEZ CABRERO, G. (2021): "El impacto de la Covid-19 en la población mayor dependiente en España con especial referencia al sector residencial", Ciencia \& Saude coletiva, enero 2021, pendiente de publicación.

MISHRA, R. (1996): “The Welfare of Nations", en BOYER, R. y DRACHE, D. (eds.), States Against Markets, Routledge, Londres.

RODRÍGUEZ CABRERO, G. (2004): El Estado de Bienestar en España: debates, desarrollo y retos, Editorial Fundamentos, Madrid.

TORTOSA CHULIÁ, M.A.; FUENMAYOR FERNÁNDEZ, A. y GRANELL PÉREZ, R. (2017): “Instrumentos de financiación y gestión en residencias de personas mayores", Informes Envejecimiento en red, $\mathrm{n}^{0}$ 16, p. 61, 〈http://envejecimiento. csic.es/documentos/documentos/enredfinanresi-16.pdf〉.

ZALAKAIN, J.; DAVEY, V; y SUÁREZ-GONZÁLEZ, A. (2020): "The COVID-19 on users of Long Term Care services in Spain", LTCcovid: International Long Term Care Policy Network, CPEC-LSE, 〈https:// Itccovid.org/wp-content/uploads/2020/05/ LTCcovid-Spain-country-report-28-May-1.pdf〉. 
La base de datos procede de la Muestra Continua de Vidas Laborales (MCVL) de la Seguridad Social, que ofrece información de los afiliados desde una perspectiva multidimensional: datos personales, tipos de contratos, días de alta en cada contrato, bases de cotización, tipo de organización, entre otros. Se enlazan las características de cada individuo de la muestra con la identidad jurídica del empleador (empresa mercantil, organización no lucrativa, etc.), y el CNAE ${ }^{14}$ al que está adscrita la empresa/organización. Para realizar el estudio cuyos datos se muestran en este artículo se toma el CNAE 873, que identifica a los establecimientos residenciales para mayores y con discapacidad física.

La MCVL no ofrece datos desglosados entre establecimientos residenciales para mayores (CNAE 8731) y establecimientos residenciales para personas con discapacidad física (CNAE 8732) ya que solo proporciona información desagregada como máximo a nivel de tres dígitos. Se toma como hipótesis que las características laborales y el número estimado de la población son representativas de la de los empleados en los establecimientos residenciales para mayores ya que estos representan la mayoría sobre el total de ambos tipos de centros.

El registro de personas con estas características se recoge de la MCVL de los años 2012, 2015 y 2018, pudiéndose construir un conjunto de datos homogéneos para cada uno de los años. Las cifras se contrastan con otras estadísticas siempre que sean accesibles. Los resultados en valores relativos son representativos del sector objeto de estudio. Los resultados se muestran para cada uno de los años analizados. La muestra es representativa a nivel estatal y a nivel de comunidades autónomas.

${ }^{14}$ El CNAE (Clasificación Nacional de Actividades Económicas) es un sistema de numeración cuya finalidad es agrupar y clasificar todas las unidades productoras según la actividad económica que desarrollan. A cada unidad se le asigna un código numérico, y a este se le llama Código CNAE. 